\title{
Downregulation of thymidylate synthase by RNAi molecules enhances the antitumor effect of pemetrexed in an orthotopic malignant mesothelioma xenograft mouse model
}

\author{
AMR S. ABU LILA ${ }^{1,4}$, CHIHIRO KATO $^{1}$, MASAKAZU FUKUSHIMA ${ }^{2}$, \\ CHENG-LONG HUANG ${ }^{3}$, HIROMI WADA ${ }^{3}$ and TATSUHIRO ISHIDA ${ }^{1,2}$
}

\begin{abstract}
Departments of ${ }^{1}$ Pharmacokinetics and Biopharmaceutics, ${ }^{2}$ Cancer Metabolism and Therapy, Institute of Health Biosciences, Tokushima University, Tokushima; ${ }^{3}$ Department of Thoracic Surgery, Faculty of Medicine, Kyoto University, Kyoto, Japan;

${ }^{4}$ Department of Pharmaceutics and Industrial Pharmacy, Faculty of Pharmacy, Zagazig University, Zagazig, Egypt
\end{abstract}

Received December 1, 2015; Accepted January 5, 2016

DOI: 10.3892/ijo.2016.3367

\begin{abstract}
Malignant pleural mesothelioma (MPM) is an incurable cancer with an increasing incidence. Currently, pemetrexed (PMX)-based chemotherapy is the mainstay of chemotherapy for MPM, however, the outcome of PMX-based chemotherapy in patients with MPM is dismal. RNA interference (RNAi) technology has been considered as an effective tool to substantially enhance the therapeutic efficacy of chemotherapeutic agents in many preclinical and clinical settings. In this study, therefore, we investigated whether non-viral anti-thymidylate synthase RNAi embedded liposome (TS shRNA lipoplex) would effectively guide the downregulation of TS in human malignant mesothelioma MSTO-211H cells. Consequently, it enhanced the antitumor effect of PMX both in vitro and in vivo. TS shRNA effectively enhanced the in vitro cell growth inhibition upon treatment with PMX via downregulating TS
\end{abstract}

Correspondence to: Dr Tatsuhiro Ishida, Department of Pharmacokinetics and Biopharmaceutics, Institute of Health Biosciences, Tokushima University, 1-78-1 Sho-machi, Tokushima 770-8505, Japan

E-mail: ishida@tokushima-u.ac.jp

Abbreviations: CHOL, cholesterol; DC-6-14, O,O'-ditetradecanoyl$\mathrm{N}$-( $\alpha$-trimethyl ammonioacetyl) diethanolamine chloride; DiR, 1,1'-dioctadecyl-3,3,3',3'-tetramethyl indotricarbocyanine iodide; DOPC, dioleoylphosphatidylcholne; DOPE, dioleoylphosphatidylethanolamine; DHFR, dihydrofolate reductase; EGFR, epidermal growth factor receptor; FBS, fetal bovine serum; GAPDH, glyceraldehydes-3-phosphate dehydrogenase; GARFT, glycinamide ribonucleotide formyltransferase; ILS, increased life span; IVIS, in vivo imaging system; MPM, malignant pleural mesothelioma; MST, mean survival time; MTT, 3-(4,5-dimethylthiazol-2-yl)-2,5diphenyltetrazolium bromide; PBS, phosphate buffer saline; PMX, pemetrexed; ROI, region of interest; TS, thymidylate synthase

Key words: lipoplex, mesothelioma, pemetrexed, short hairpin RNA, thymidylate synthase expression in the MSTO-211H cell line. In in vivo orthotopic tumor model, the combined treatment of PMX and TS shRNA lipoplex efficiently combated the progression of orthotopic thoracic tumors and as a result prolonged mouse survival, compared to each single treatment. Our findings emphasize the pivotal relevance of RNAi as an effective tool for increasing the therapeutic efficacy of PMX, a cornerstone in the treatment regimens of MPM, and thereby, raising the possibility for the development of a novel therapeutic strategy, combination therapy of TS-shRNA and PMX, that can surpass many of the currently applied, but less effective, therapeutic regimens against lethal MPM.

\section{Introduction}

Malignant pleural mesothelioma (MPM) is an aggressively growing neoplasm, which disseminates into the thoracic cavity and frequency produces a malignant pleural effusion $(1,2)$. MPM is most often caused by asbestos exposure with a long latency period, often exceeding 20 years between the first exposure to asbestos and a diagnosis of the disease $(3,4)$. Despite the fact that this type of tumor was once considered rare, the current incidence is predicted to increase globally and peak in the coming decades particularly in developing countries where the use of asbestos is still prevalent $(5,6)$.

Many therapeutic modalities have been applied for the treatment of MPM including surgery, radiotherapy and chemotherapy (7). Chemotherapy is considered the main therapeutic option for patients with both unresectable and resectable tumors. Chemotherapy alone or when used in combination with other adjuvant therapeutic modalities such as radiotherapy, might be the only therapeutic modality currently in use for MPM $(8,9)$. Nevertheless, chemotherapeutic regimens used against MPM have not proven effective to date, because MPM is often resistant to chemotherapy $(10,11)$.

Pemetrexed (PMX), an anti-folate agent, is one of the currently approved chemotherapeutic agents for the first line care of patients with MPM $(12,13)$. It acts mainly via inhibiting the multiple folate-dependent enzymes that are involved 
in DNA synthesis and repair: thymidylate synthase (TS), dihydrofolate reductase (DHFR), and glycinamide ribonucleotide formyltransferase (GARFT) $(14,15)$. However, PMX has shown only modest activity in patients with MPM either when used alone or in combination with other chemotherapeutic agents such as cisplatin (16). Many studies have reported that a high expression of TS, the rate-limiting enzyme of de novo DNA synthesis, can significantly predict both poor sensitivity and resistance to PMX-based chemotherapy $(17,18)$. Therefore, the development of strategies to downregulate the expression of TS is anticipated to enhance the cytotoxic efficacy of PMX against MPM, and, thus, improve the therapeutic outcome in patients treated with PMX.

RNA interference (RNAi), a novel regulatory process in which double-stranded RNA (dsRNA) induces a specific degradation of its target mRNA, has rapidly become a highly specific and powerful tool to silence target genes (19-21). Many studies have revealed that RNAi can be a novel tool for clarifying gene function and applicable to gene-specific therapeutics (22-24). In the present study, therefore, we assumed that downregulation of the TS gene by RNAi might be efficient in enhancing the chemosensitivity of MPM cells to PMX. To validate our assumption, we evaluated the efficacy of a TS gene knockdown via a chemically synthesized short hairpin RNA (shRNA) designed against TS to enhance the cytotoxicity of PMX against the human mesotheliomal cell line MSTO-211H, in vitro. In addition, we evaluated the in vivo antitumor efficacy of a combination therapy with TS-shRNA lipoplex and PMX in an orthotopic xenograft mouse model.

\section{Materials and methods}

Materials. Pemetrexed disodium (PMX; Alimta ${ }^{\circledR}$ ) was purchased from Eli Lilly (Indianapolis, IN, USA). Dioleoylphosphatidylcholine (DOPC) and dioleoyl-phosphatidylethanolamine (DOPE) were generously donated by NOF Inc. (Tokyo, Japan). Cholesterol (CHOL) and D-luciferin potassium salt were purchased from Wako Pure Chemical (Osaka, Japan). 1,1'-Dioctadecyl-3,3,3',3'-tetramethyl indotricarbo-cyanine iodide (DiR) was purchased from Invitrogen (OR, USA). A cationic lipid, O,O'-ditetradecanoyl-N- $(\alpha-$ trimethyl ammonioacetyl) diethanolamine chloride (DC-6-14) was purchased from Sogo Pharmaceutical (Tokyo, Japan). 3-(4,5-dimethylthiazol-2-yl)-2,5-diphenyltetrazolium bromide (MTT) was purchased from Nacalai Tesque (Kyoto, Japan). All other reagents were of analytical grade.

Animals and tumor cell line. 5-week-old male BALB/c $n u / n u$ mice were purchased from Japan SLC (Shizuoka, Japan). All animal experiments were evaluated and approved by the Animal and Ethics Review Committee of Tokushima University (permission No. 13086). A human malignant pleural mesothelioma (MPM) cell line, MSTO-211H expressing firefly luciferase (MSTO-211H-Luc) generated by stable transfection with the firefly luciferase gene (pGL3 Basic plasmid; Promega, Madison, WI, USA) was generously supplied by Dr Masashi Kobayashi, Department of Thoracic Surgery, Faculty of Medicine, Kyoto University (Kyoto, Japan) and was maintained in RPMI-1640 medium (Wako Pure Chemical).
Chemically synthesized shRNAs. All shRNAs, chemically synthesized and purified by high performance liquid chromatography, were purchased from Hokkaido System Science (Sapporo, Japan). The sequence of shRNA against thymidylate synthase (TS shRNA) was 5'-GUAACACCAUCGAUCAU GAUAGUGCUCCUGGUUGUCAUGAUCGAUGGUGUUA CUU-3' and for a nonspecific shRNA nonspecific (NS shRNA, not to target any gene in either the human or mouse genome) was 5'-CUUAAUCGCGUAUAAGGCUAGUGCUCCUGGU UGGCCUUAUACGCGAUUAAGAUU-3'. shRNAs were dissolved in RNase-free TE buffer at a final concentration of $100 \mathrm{nmol} / \mathrm{ml}$.

shRNA transfection. MSTO-211H cells were transfected with shRNA using Lipofectamine ${ }^{\circledR}$ RNAiMAX (LfRNAiMAX, Invitrogen, Carlsbad, CA, USA) according to the manufacturer's instructions. Briefly, 300 pmol shRNA and $15 \mu \mathrm{l}$ LfRNAiMAX were diluted in Opti-MEM I (Invitrogen) to a total volume of $500 \mu \mathrm{l}$. The diluted shRNA and LfRNAiMAX were mixed and incubated at room temperature for $20 \mathrm{~min}$ to form the shRNA/LfRNAiMAX complex. The shRNA/ LfRNAiMAX complex was then applied to the cells followed by incubation for the indicated time interval.

Real-time quantitative reverse transcriptase ( $q R T)$-PCR analysis. RNA isolation and cDNA synthesis were performed according to the manufacturer's instructions. Briefly, in 6 -well plates, $5 \times 10^{4}$ cells were seeded for $24 \mathrm{~h}$ before shRNA transfection. The cells were transfected with 5 or $10 \mathrm{nM}$ of either TS shRNA or NS shRNA, as described above. At 72-h post-transfection, the total RNA of the MSTO-211H cells was isolated using an RNaqueous-micro kit (Ambion, Austin, TX, USA). To conduct the reverse transcription reaction, $2 \mu \mathrm{l}$ of RNA was converted to cDNA with a total volume of $20 \mu \mathrm{l}$, including $500 \mathrm{nM}$ of Oligo(dT)20, $500 \mu \mathrm{M}$ dNTP, $1 \mu 1$ of RNase inhibitor, and $1 \mu 1$ of ReverTra Ace (Toyobo, Osaka, Japan). Real-time PCR was performed on a StepOnePlus real-time PCR system (Applied Biosystems, CA, USA) with a FastStart TaqMan Probe Master and Universal ProbeLibrary (Roche Diagnostics GmbH, Manheim, Germany) according to the manufacturer's instructions. The TS primers and a probe were from the Assay-on-Demand gene expression assay mix (TS assay ID Hs00426591-ml, PCR product size 87 bp; Applied Biosystems). The GAPDH primers and a probe were designed using ProbeFinder software (Roche Diagnostics GmbH). The GAPDH primers and the probes were as follows: GAPDH primers and probe (forward 5'-GCTCTCTGCTCCTCCTGTTC-3' and reverse 5'-ACGACCAAATCCGTTGACTC-3', probe \#60). The amplification conditions were as follows: $10 \mathrm{~min}$ at $95^{\circ} \mathrm{C}$, followed by 40 cycles at $95^{\circ} \mathrm{C}$ for $15 \mathrm{sec}$ and at $60^{\circ} \mathrm{C}$ for $1 \mathrm{~min}$. The results were expressed as the threshold cycle $\left(\mathrm{C}_{\mathrm{T}}\right)$, and the relative expression levels for each primer set were normalized to the internal control expression of GAPDH using the $2^{-\Delta \Delta C T}$ method. The TS mRNA expression level of non-transfected cells was set at $100 \%$. Three independent experiments were performed with the same results.

In vitro cytotoxicity assay. MSTO-211H cells were seeded onto 96-well plates at a density of 2,000 cells per well $24 \mathrm{~h}$ before 
shRNA transfection. The cells were transfected with $5 \mathrm{nM}$ of either TS shRNA or NS shRNA for $24 \mathrm{~h}$. After transfection, the culture medium was replaced with fresh medium containing various concentrations of PMX, range: 0.001-1,000 ng/ml. Following $72 \mathrm{~h}$ incubation at $37^{\circ} \mathrm{C}$, media were discarded and MTT assay was conducted as described previously (25). Cell viability (\%) was calculated from the ratio between the percentage of viable treated cells and viable untreated cells.

Preparation of cationic liposomes. Cationic liposome composed of DOPE:DOPC:DC-6-14 (3:2:5 molar ratio) was prepared as previously described (23). To follow the in vivo distribution of shRNA lipoplexes, $0.1 \mathrm{~mol} \%$ of the fluorescent dye DiR was incorporated in the lipid mixture. The mean diameter and zeta potential for cationic liposomes was $102.8 \pm 32.7 \mathrm{~nm}$ and $49.1 \pm 3.7 \mathrm{mV}(\mathrm{n}=3)$, respectively, as determined with a NICOMP 370 HPL submicron particle analyzer (Particle Sizing System, Santa Barbara, CA, USA). The concentration of phospholipids was determined by colorimetric assay (26).

Preparation of shRNA lipoplexes. For the preparation of shRNA/cationic liposome complex (shRNA lipoplex), shRNA and cationic liposome were mixed at a molar ratio of 2000/1 (lipid/shRNA, molar ratio), and the mixture was vigorously vortexed for $10 \mathrm{~min}$ at room temperature to form shRNA lipoplex. The mean diameter and zeta potential of the shRNA lipoplex was $395.3 \pm 32.2 \mathrm{~nm}$ and $30.6 \pm 1.9 \mathrm{mV}(\mathrm{n}=3)$, respectively. To detect the absence of free-shRNA in the prepared shRNA lipoplex, electrophoresis was performed on $2 \%$ agarose gel.

Intrapleural orthotopic implantation model. For the development of the orthotopic implantation model, five-week-old male BALB/c nu/nu mice were anaesthetized with 2,2,2-tribromoethanol (Avertin; Sigma-Aldrich) and injected directly into the left pleural cavity with $1 \times 10^{6}$ MESTO-211H-Luc cells in $100 \mu \mathrm{l}$ of PBS. IVIS was used to monitor the development of thoracic tumors. When establishment of thoracic tumors was ensured, the mice were randomized into control and treatment groups ( $n=6 /$ group).

In vivo distribution study of DiR-labeled lipoplex. To follow the in vivo distribution of lipoplex in orthotopic tumor-bearing mice $(n=5)$, mice were intrapleurally injected with $50 \mu 1$ of DiR-labeled shRNA lipoplex (20 $\mu \mathrm{g}$ shRNA/mouse). At selected post-injection time points (5 and $30 \mathrm{~min} ; 1,2,6,12$, 24, 48, 72, 96 and $120 \mathrm{~h}$ ), mice were anesthetized by isoflurane and the in vivo distribution of DiR-labeled shRNA lipoplex was visualized using an in vivo imaging system (IVIS, Xenogen, CA, USA). The fluorescence images were acquired using an exposure time of $1 / 8 \mathrm{sec}$. For the ex vivo fluorescence imaging analyses, five major organs (liver, spleen, lung, kidney and heart) were harvested after the final measurement and immediately subjected to fluorescence imaging using the IVIS imaging system.

Therapeutic efficacy in an orthotopic tumor mouse model. For the therapeutical experiments, at day 7 post-tumor cells implantation, when the establishment of pleural metastasis was ensured, the mice were divided into 6 groups $(n=6)$ : a control group treated with sucrose and 5 groups treated with either NS shRNA lipoplex, TS shRNA lipoplex, free PMX, NS shRNA lipoplex + free PMX, or TS shRNA lipoplex + free PMX. In groups treated with shRNA lipoplex, mice were intrathoracically injected with shRNA lipoplex (20 $\mu \mathrm{g} / \mathrm{mouse})$ on days 7 , 9, 11, 13, 15 and 17. In the groups treated with free PMX, PMX $(25 \mathrm{mg} / \mathrm{kg}$ ) was intraperitoneally (i.p.) administered on days 7 , $8,9,10,11,14,15,16,17$ and 18 after tumor cell implantation. The antitumor efficacy was evaluated in terms of both the mean survival time (MST) and the percentage of increased life span [ILS (\%)]. The MST (day) was identified by recording the mortality on a daily basis for 45 days, and the ILS (\%) was calculated using the following equations: MST (day) = day of the first death + day of the last death/2 (27); ILS (\%) $=[($ mean survival time of treated group/mean survival time of control group) -1] x100 (28).

Bioluminescence imaging with IVIS. Seventy-two hours after the last treatment (on day 21), the mice were anesthetized with isoflurane inhalation, and were subsequently i.p.injected with $100 \mu \mathrm{l}$ of $7.5 \mathrm{mg} / \mathrm{ml}$ D-luciferin potassium salt. Bioluminescence in vivo imaging was initiated $5 \mathrm{~min}$ after injection and bioluminescence from the region of interest (ROI) was defined manually. Background photon-flux was defined using a ROI from a mouse that was not given an i.p. injection of D-luciferin potassium salt. All bioluminescent data were collected and analyzed using IVIS. Following in vivo imaging procedures, the mice were euthanized, the thoracic tumors were carefully removed, and the in vivo gene knockdown effect of treatments was determined by qRT-PCR as described above.

Statistical analysis. All values are expressed as the mean \pm SD. Statistical analysis was performed with a two-tailed unpaired Student's t-test and one way ANOVA using GraphPad InStat View software (GraphPad Software, San Diego, CA, USA). The level of significance was set at $\mathrm{p}<0.05$.

\section{Results}

In vitro downregulation of TS $m R N A$ expression by chemically synthesized shRNA. To quantify the gene knockdown efficiency, MSTO-211H cells were transfected with either 5 or $10 \mathrm{nM}$ TS shRNA or NS shRNA for $72 \mathrm{~h}$, then the mRNA expression levels of TS were quantified using qRT-PCR. Compared with untransfected cells, the mRNA expression levels of TS were downregulated $>90 \%(\mathrm{p}<0.001)$ in the TS shRNA transfected cells (Fig. 1). The NS shRNAtransfected cells exhibited no significant change in TS mRNA expression compared with untreated cells.

In vitro cell growth inhibition by a combination of TS ShRNA and PMX treatment. To investigate whether TS gene knockdown affects the in vitro cytotoxicity of PMX against MSTO-211H cells, cell viability after exposure to PMX alone, or to a combination of TS shRNA and PMX, was evaluated via MTT assay. As shown in Fig. 2, PMX treatment decreased cell viability in a dose-dependent manner. In addition, the combined treatment with NS shRNA and PMX was as toxic as 


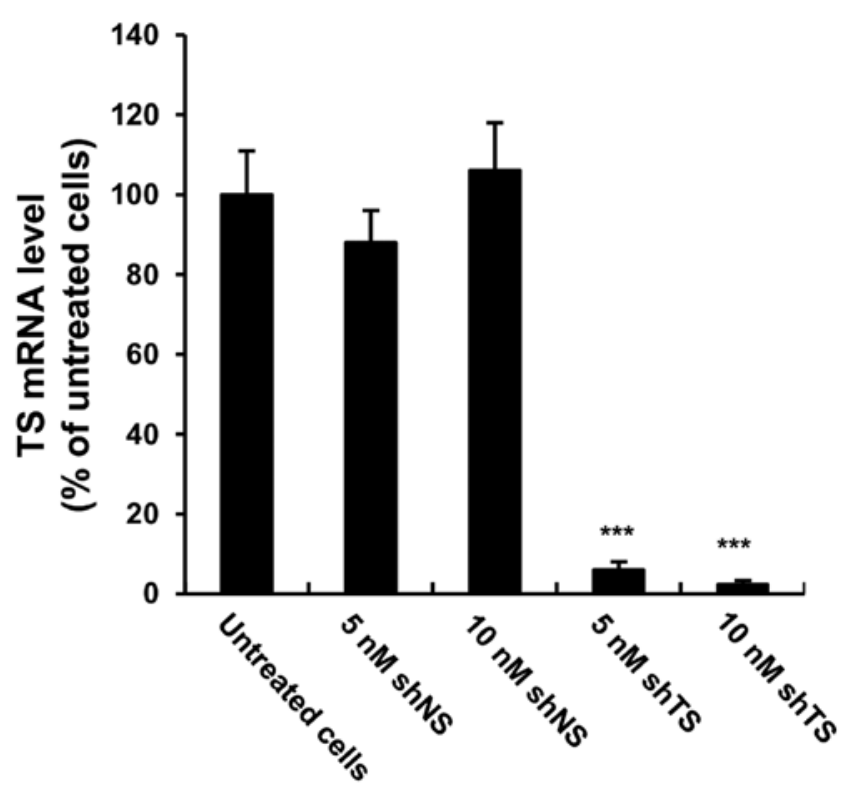

Figure 1. Suppression of TS gene expression by in vitro transfection of TS shRNA in MSTO- $211 \mathrm{H}$ cells. MSTO-211H cells were transfected with either NS shRNA or TS shRNA (5 and $10 \mathrm{nM}$ ) using Lf RNAiMAX. At $72 \mathrm{~h}$ post-transfection, total RNA in the cells was extracted and the mRNA levels were quantified using qRT-PCR. Data represent the mean \pm SD from three independent experiments. ${ }^{* * *} \mathrm{p}<0.001$ compared with untreated cells.

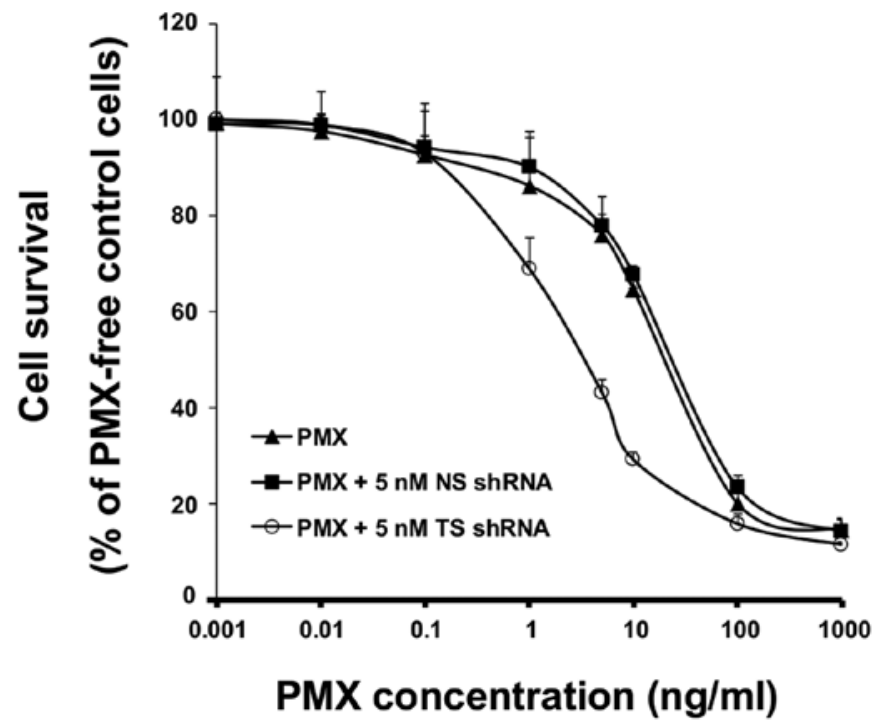

Figure 2. Effect of combined TS silencing and treatment with PMX on the cellular viability of MSTO-211H cells. MSTO-211H cells, untreated or treated with either $5 \mathrm{nM}$ TS shRNA or NS shRNA, were incubated with various concentrations of PMX $(0.001$ to $1000 \mathrm{ng} / \mathrm{ml})$ for $72 \mathrm{~h}$ at $37^{\circ} \mathrm{C}$. Cell viability was determined by MTT assay. Data represent the mean $\pm \operatorname{SD}(n=3)$.

PMX alone, whereas the combination of TS shRNA with PMX resulted in a significantly higher cytotoxicity than that caused by PMX alone $\left(\mathrm{IC}_{50} 2.74 \pm 0.20 \mathrm{ng} / \mathrm{ml}\right.$ versus $38.82 \pm 1.70 \mathrm{ng} / \mathrm{ml}$, respectively) $(\mathrm{p}<0.05)$, suggesting the occurrence of a synergistic effect between TS shRNA and PMX.

In vivo antitumor efficacy of the combined therapy of $T S$-shRNA and PMX in an orthotopic implantation model. To examine the effect of the combined treatment of TS shRNA
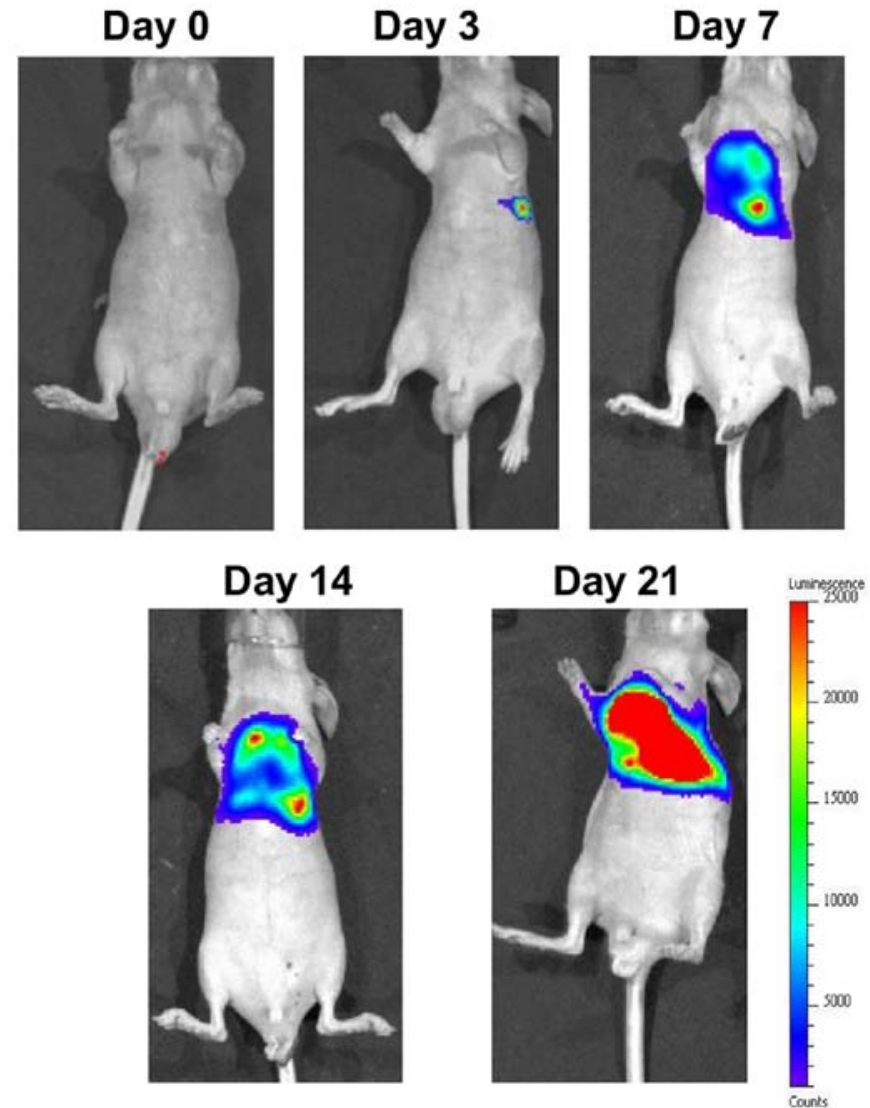

Figure 3. Bioluminescent monitoring of thoracic tumor establishment. MSTO-211H-Luc cells $\left(1 \times 10^{6}\right)$ were injected into the left thoracic cavity of the mice $(n=6)$. The establishment of thoracic tumors was monitored at different time intervals (on 0,3, 7, 14 and 21 days after tumor inoculation) using a bioluminescent in vivo imaging system (IVIS). All bioluminescent images were acquired with a $30 \mathrm{sec}$ exposure time. The picture represents typical examples from 6 implanted mice.

and PMX on tumor growth in vivo, we implanted MSTO$211 \mathrm{H}-\mathrm{Luc}$ into the left thoracic cavity of nude mice and treatment was started at day 7 post-tumor inoculation; the time at which thoracic tumors were visible (Fig. 3). At $72 \mathrm{~h}$ after the last treatment (on day 21), the bioluminescence related to tumor progression was monitored by IVIS (Fig. 4). TS shRNA alone and PMX alone and a combination of NS shRNA plus PMX all exerted a moderate degree of inhibitory effect on the development of thoracic tumors, as indicated by a slight reduction in tumor burden (Fig. 4A), and, consequently, a slight decrease in photon intensity, compared with the control group (Fig. 4B). On the contrary, treatment with a combined therapy of TS shRNA and PMX significantly inhibited the production of thoracic tumors, which was indicated by a remarkable reduction in tumor burden (Fig. 4A) and a substantial decrease in the photon intensity, compared with the other treated groups $(\mathrm{p}<0.001)$ (Fig. 4B). The dose and schedule of the combined treatment of TS-shRNA and PMX were well tolerated, as determined by the absence of a significant amount of weight loss or other signs of acute or delayed toxicity (data not shown).

Mouse survival was evaluated up to 45 days after a xenograft injection of MSTO-211H-Luc. The survival of treated mice is illustrated in Fig. 4C, and the mean survival time [MST 


\section{A}
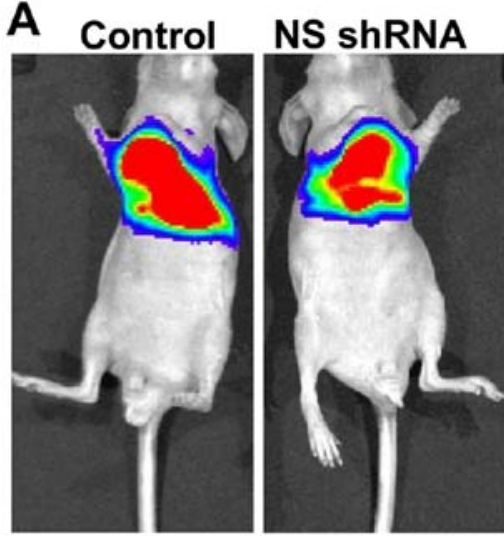

PMX

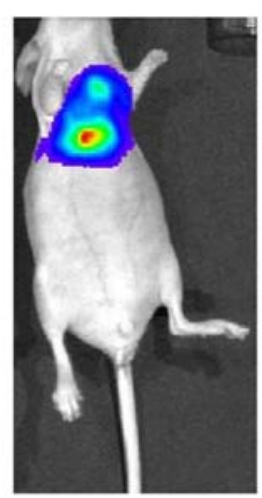

\begin{abstract}
PMX + NS
\end{abstract} ShRNA
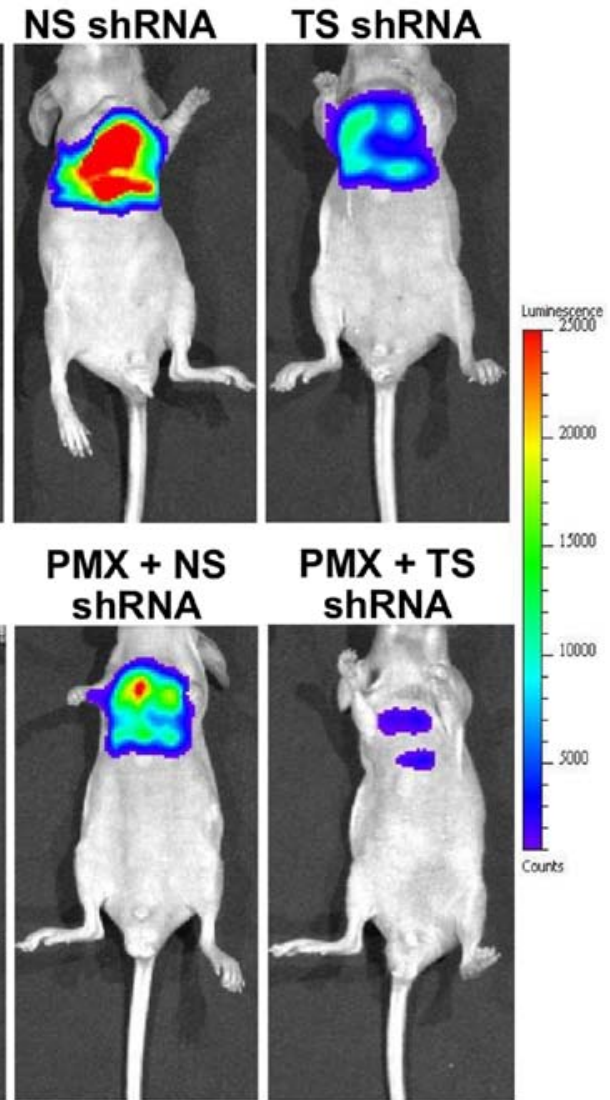

Figure 4. Effect of the combined treatment of PMX and TS shRNA on the production of thoracic tumors. MSTO-211H-Luc cells $\left(1 \times 10^{6}\right)$ were injected into the left thoracic cavity of the mice. Seven days later, mice were randomized into 6 groups $(n=6)$ : a control group receiving 9\% sucrose and 5 treatment groups. PMX (25 mg/kg) was intraperitoneally administered daily for 5 consecutive days/week for 2 weeks. shRNA lipoplex containing either nonspecific (NS) shRNA or TS shRNA (20 $\mu \mathrm{g}$ shRNA/mouse) was intrapleurally injected every other day from day 7 to day 17 after tumor cell inoculation. (A) Monitoring the growth of MSTO-211H-Luc xenografts by IVIS. Representative mouse tumor imaging was performed $72 \mathrm{~h}$ after the end of treatment (on day 21). The picture represents a typical example from the 6 mice. (B) Quantitative analysis of the bioluminescence photon counts were used as a measure of tumor growth. (C) The mortality was recorded on a daily basis for 45 days. Data are reported as the mean \pm SD $(n=4) .{ }^{*} p<0.05$, ${ }^{* * *} \mathrm{p}<0.01$ and $^{* * * *} \mathrm{p}<0.001$ versus control.

(day)] and percentage of increased life span [ILS (\%)] are summarized in Table I. There was no significant difference in survival between the control and the NS shRNA-treated groups (Fig. 4C). Treatment with either as single agents slightly prolonged the survival, compared with the control group [ILS (\%) were 20.0 and $26.6 \%$, respectively], whereas, the combined treatment of TS shRNA lipoplex or PMX showed a significant survival advantage; $50 \%$ of the mice (3 out of 6 ) became long-term survivors ( $>45$ days) (Fig. 4C), compared with other treated groups.

In vivo gene silencing efficacy of the combined therapy of TS-shRNA and PMX in an orthotopic implantation model. To gain further insights into the in vivo gene silencing efficacy of TS shRNA alone or combined with PMX, the TS mRNA levels were measured quantitatively via RT-PCR in the thoracic tumors $72 \mathrm{~h}$ after the last treatment (on day 21). Compared with untreated (control) mice or mice treated with NS shRNA
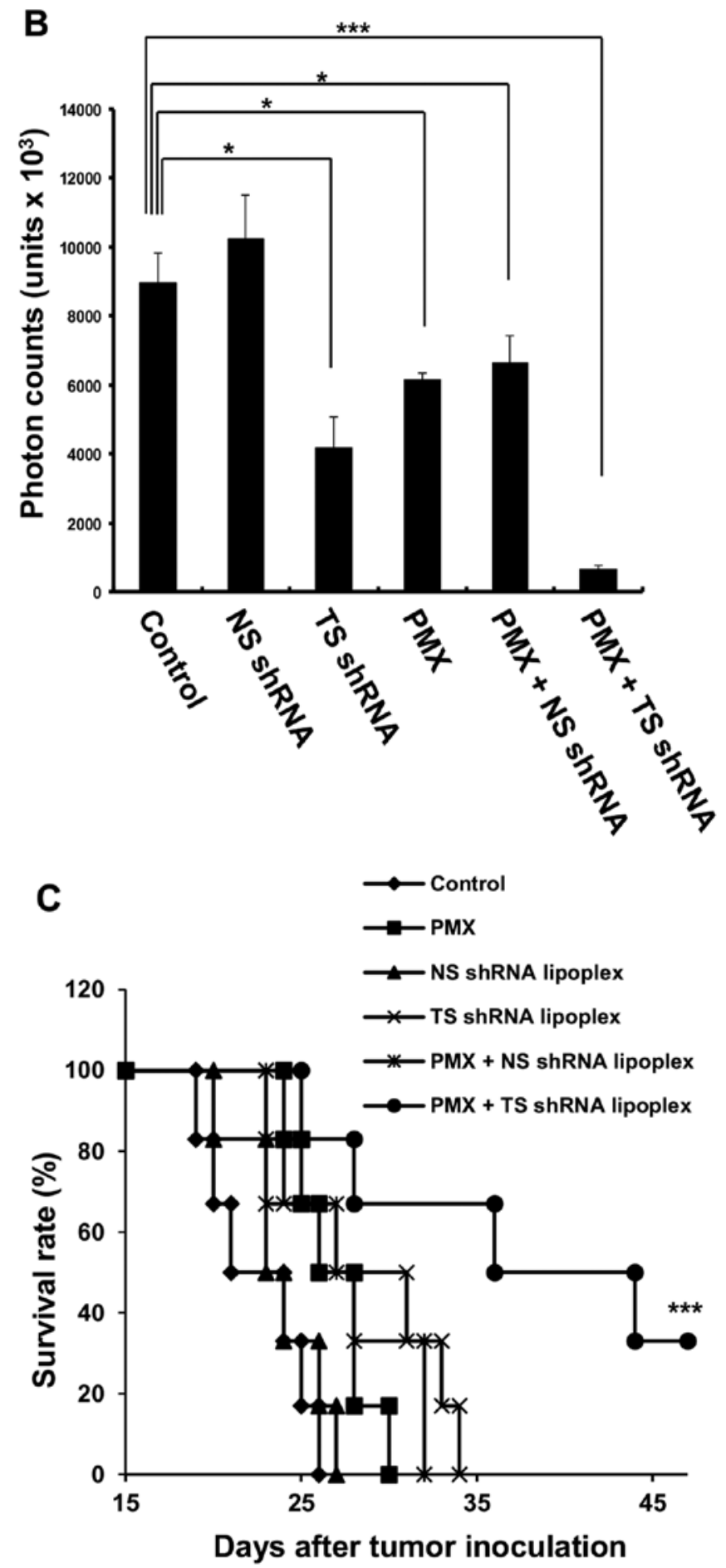

lipoplex, a significant reduction in the TS mRNA level was observed in mice treated with either, reaching approximately 30 and 50\%, respectively (Fig. 5). Unfortunately, the amount of total RNA isolated from thoracic tumors treated with a combination treatment of TS shRNA lipoplex or PMX was too low to determine the TS mRNA level. These results emphasize the contribution that in vivo gene silencing of TS shRNA made to the superior antitumor efficacy of the combined treatment of PMX plus TS shRNA lipoplex.

In vivo biodistribution study following intrapleural administration of shRNA lipoplex. The efficient delivery 
Table I. Summary of median survival time (MST) and percent increased life span [ILS (\%)] of tumor-bearing mice after treatment with PMX and/or shRNAs.

\begin{tabular}{lcc}
\hline Formulation & MST (days) & ILS (\%) \\
\hline Control (9\% sucrose solution) & 22.5 & - \\
NS shRNA lipoplex & 24.0 & 6.6 \\
TS shRNA lipoplex & 28.5 & 26.6 \\
PMX & 27.0 & 20.0 \\
PMX + NS shRNA lipoplex & 27.5 & 22.2 \\
PMX + TS shRNA lipoplex & $>39$ & $>75.0$ \\
\hline
\end{tabular}

Data were obtained from Fig. 4C. MST and ILS (\%) were calculated with the equations described in Materials and methods.

and prolonged retention of shRNA lipoplex in the target tissues are key determinants for the therapeutic efficacy of shRNA. Therefore, we investigated the in vivo distribution of fluorescent-labeled lipoplex following intrapleural injection in tumor-bearing mice (Fig. 6). DiR was applied into the liposomal membrane as the hydrophobic fluorescent probe since its near-infrared excitation and emission wavelengths could efficiently reduce the interference of animal auto-fluorescence (29). In the mice bearing thoracic tumors, shRNA lipoplex were retained within the pleural cavity for an extended period (120-h post-injection) (Fig. 6A). No remarkable accumulation of shRNA lipoplex in the major organs, particularly the liver and spleen, was observed following the intrapleural injection of shRNA lipoplex into orthotopic tumor-bearing mice, as presented in the ex vivo images (Fig. 6B). It appears that orthotopic implantation of MSTO$211 \mathrm{H}$ cells within the pleural cavity confined the shRNA lipoplexes to the thoracic cavity. These results suggest that the significant prolonged retention of shRNA lipoplex within the pleural cavity of orthotopic tumor-bearing mice contributed to the enhanced therapeutic efficacy of shRNA lipoplex following intrapleural administration.

\section{Discussion}

Pemetrexed (PMX) represents a mainstay in malignant pleural mesothelioma (MPM) treatment, and resistance to its activity is a major obstacle to successful chemotherapy for MPM. Resistance to PMX has been attributed mainly to an increased expression of the thymidylate synthase (TS) enzyme, a key enzyme for DNA synthesis and repair. Many reports have investigated the clinical relevance of high TS mRNA as a predictor of the resistance to PMX in various cancers including MPM (30-32). Therefore, downregulation of the TS enzyme is considered an attractive strategy that could enhance MPM therapy with anti-folate agents including PMX. In the present study, we proposed a novel RNAi approach that uses a chemically synthesized short hairpin RNA (shRNA) to downregulate the TS gene and thus restore the chemosensitivity of PMX against human MPM (MSTO-211H) cells both in vitro and in vivo. We demonstrated that RNAi knockdown of the TS gene in MSTO-211H cells suppressed the expression

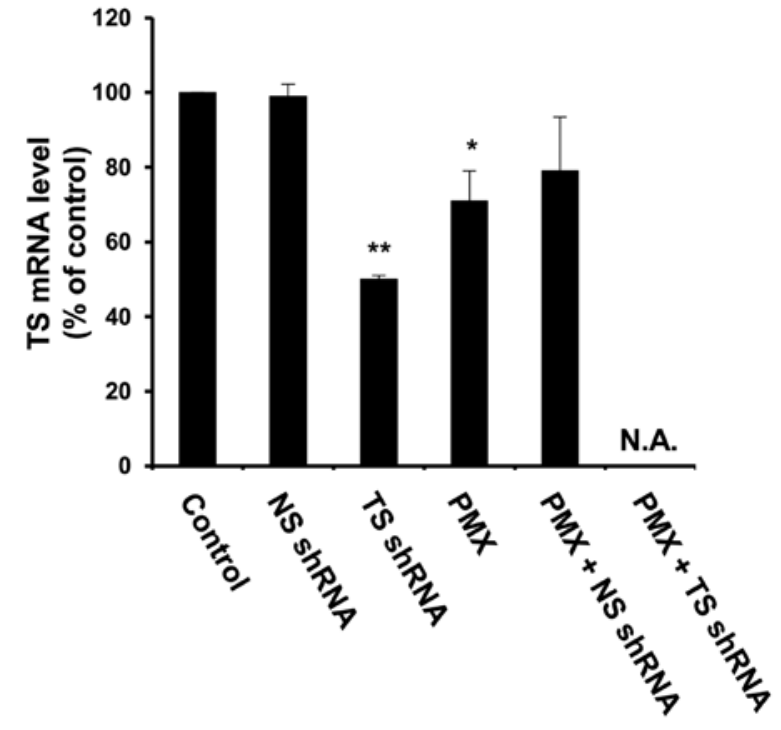

Figure 5. Suppression of TS mRNA expression in the tumor tissue after in vivo treatment. Thoracic tumors derived from mice treated as indicated in the legend of Fig. 3 were subjected to total RNA isolation. Then, the TS mRNA levels were determined via qRT-PCR. Data represents the mean \pm SD. ${ }^{*} \mathrm{p}<0.05$ and ${ }^{* * *} \mathrm{p}<0.001$ versus control.

of TS mRNA by approximately $90 \%$ (Fig. 1), and resulted in improved chemosensitivity to PMX in vitro (Fig. 2). In addition, intrapleural administration of TS shRNA lipoplex in combination with PMX significantly suppressed the growth of thoracic tumors in orthotopic tumor-bearing mouse models (Fig. 4), which resulted in an increase in the life span of treated mice (Table I). These results emphasize the pivotal relevance of RNAi as an effective tool for increasing the therapeutic efficacy of PMX against human MPM.

RNA interference (RNAi), a sequence-specific gene silencing mechanism induced by double-stranded RNA (dsRNA), has emerged as a promising therapy for combating cancer $(19,33,34)$. The RNAi effect can be mediated with short interfering RNA (siRNA), which is a short, double stranded RNA with 21-23 base pairs (35), or, as recently demonstrated, with short hairpin RNA (shRNA) (36). Although siRNA and shRNA elicit comparable gene silencing effects in RNAi experiments, chemically synthesized shRNA represents significant advantages over siRNA. shRNA is produced from a single oligodeoxynucleotide that requires less up-front oligo design and synthesis, and it uses a shorter, high-yield transcription reaction. In addition, unlike siRNA, shRNA does not require the annealing of 2 RNA strands, which eliminates the potential for contamination of single-stranded RNA that can compromise its yield and integrity $(37,38)$. Finally, similar to siRNA, the stability and biological activity of shRNA can be improved via chemical modifications, along with a reduction in innate immune responses (39).

In our orthotopic implantation model with MPM cells, suppression of tumor progression and survival prolongation are the most valuable parameters that can be used to evaluate therapeutic efficiency. A potent tumor growth inhibitory effect has been observed with the combined treatment of TS shRNA and PMX (Fig. 4), and it was accompanied by a significant prolongation of survival (MST $>39$ day, ILS 
A

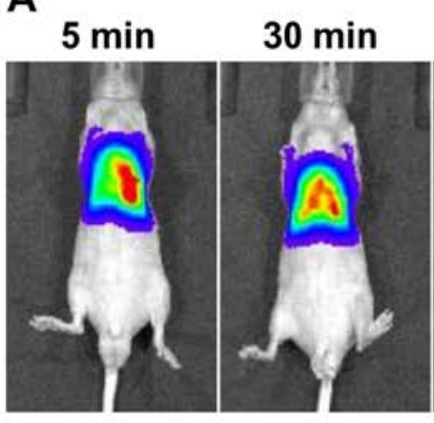

$1 \mathrm{~h}$

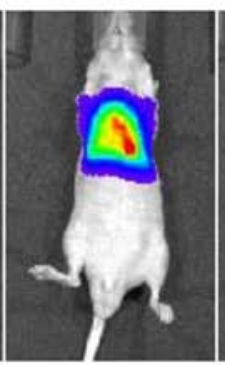

$2 \mathrm{~h}$

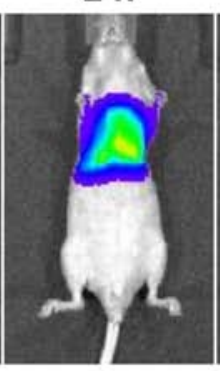

$6 \mathrm{~h}$

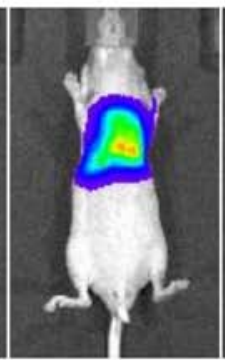

$12 \mathrm{~h}$

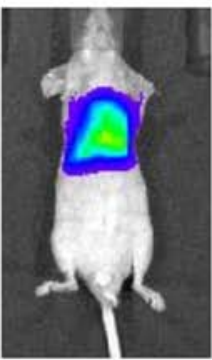

B

\section{Tumor-bearing mice}

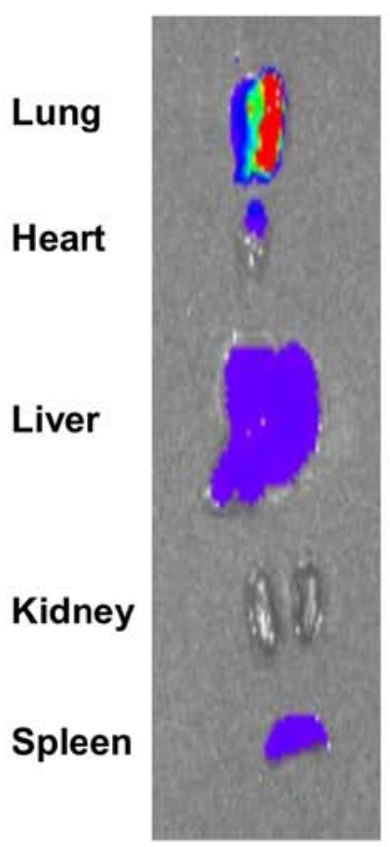

Figure 6. In vivo distribution of fluorescence-labeled shRNA lipoplex. Tumor-bearing mice (n=5) were intrapleurally injected with DiR-labeled shRNA lipoplex (20 $\mu \mathrm{g} \mathrm{shRNA/mouse).} \mathrm{At} \mathrm{selected} \mathrm{post-injection} \mathrm{time} \mathrm{points} \mathrm{(5} \mathrm{and} 30 \mathrm{~min} ; 1,2,6,12,24,48,72,96$ and 120 h), in vivo distribution of the lipoplex was visualized by an in vivo imaging system. (A) In vivo distribution of shRNA-lipoplex in orthotopic tumor-bearing mice. (B) Ex vivo fluorescence images of major organs. The picture represents typical examples from 5 independent experiments for each of the tumor-bearing mice.

$>75 \%$ ) (Table I). This emphasizes the efficacy of TS shRNA in inducing potent and enduring TS gene silencing, thereby sensitizing the mesothelioma MSTO-211H cell line to the in vivo cytotoxic effect of PMX and leading to a superior level of tumor growth suppression (Fig. 5) and a prolonged life span (Fig. 4C and Table I). Furthermore, as a single agent, either remarkably inhibited the production of thoracic tumors of the MSTO-211H cell line, as indicated by a reduction in the tumor burden (Fig. 4A), and, consequently, a decrease in photon intensity (Fig. 4B). However, both treatments showed only a marginal prolongation of survival (MST, 27 and 28.5 day, ILS, 20 and $26.6 \%$, respectively), compared with untreated mice (MST, 22.5 day) (Table I), probably as a result of aggressive re-growth of tumor cells after cessation of treatment.

Of note, an in vivo imaging study demonstrated a prolonged retention of shRNA lipoplex within the pleural cavity in tumor-bearing mice (Fig. 6). The electrostatic attraction and/or binding that the positively charged surface of shRNA lipoplex presents to the negatively charged MSTO-211H tumor cells might tend to hold the shRNA lipoplex in the thoracic cavity following its intrapleural administration. This prolonged retention of shRNA lipoplex is believed to enhance its uptake by tumor cells and to induce a potent TS gene knockdown by TS shRNA (Fig. 5), which leads to an increased sensitivity of MSTO-211H cells to the cytotoxic effect of PMX. This sensitizing effect of TS shRNA, in turn, contributed substantially to the potent antitumor efficacy of PMX against MSTO-211H cells in an orthotopic tumor mouse model (Fig. 4).

Since MPM grows locally within the thoracic cavity and rarely displays metastasis to distant sites $(24,40)$, the direct administration of novel therapeutic agents such as genetic materials should be uniquely accessible. Furthermore, direct administration of genetic materials into the pleural cavity, rather than systemic administration, should eliminate/alleviate the development of severe immune reactions and/or cytokine induction against the injected genetic materials. Many clinical trials have revealed that the intrapleural administration of therapeutic agents to patients with MPM is safe and well tolerated $(41,42)$. In our study, intrapleural administration of shRNA nanoparticles (shRNA lipoplex) was safe and well tolerated by mice as indicated by the absence of remarkable toxic effects (data not shown). In addition, intrapleural administration could guarantee the effective delivery of RNAi molecules and further reduce the adverse effects to normal organs compared with systemic administration.

One of the major obstacles in cancer therapy is the occurrence of cellular resistance to cancer chemotherapy $(23,43)$. In vitro, in vivo and clinical studies have shown that the exposure to TS inhibitor compounds, including PMX, results in acute induction of TS overexpression and thus the acute development of resistance in response to TS inhibitors (30-32). In the present study, TS gene knock-down by RNAi efficiently suppressed the expression level of TS genes both in vitro (Fig. 1) and in vivo (Fig. 5), and thus restored the chemosensitivity to PMX against MSTO-211H cells. To the best of our knowledge, this is the first report regarding the efficacy of chemically synthesized shRNA, delivered by cationic liposomes, in improving the therapeutic efficacy of PMX against human MPM.

In the present study, we showed that the RNAi effect from an intrapleural injection of shRNA designed against the TS 
gene, repressed the expression of TS mRNA in human malignant mesotheliomal MSTO-211H cells and suppressed tumor progression, thereby prolonging the survival of mice inoculated orthotopically with MSTO-211H cells. Our proposed combination therapy of TS shRNA and PMX shows therapeutic promise for the treatment of MPM via the downregulation of TS expression levels and the subsequent prevention of the development of cellular resistance that is observed with TS inhibitor compounds, including PMX, that are currently used in clinical settings.

\section{Acknowledgements}

We thank Dr G.L. Scherphof for his helpful advice in preparing this manuscript. This work was supported by the Japan Society for the Promotion of Science, Grants-in-Aid for JSPS Fellows and for Scientific Research (B) (15H04639), the Ministry of Education, Culture, Sports, Science and Technology, Japan.

\section{References}

1. Giovannetti E, Zucali PA, Assaraf YG, Leon LG, Smid K, Alecci C, Giancola F, Destro A, Gianoncelli L, Lorenzi E, et al: Preclinical emergence of vandetanib as a potent antitumour agent in mesothelioma: Molecular mechanisms underlying its synergistic interaction with pemetrexed and carboplatin. Br J Cancer 105: 1542-1553, 2011.

2. Ostroff RM, Mehan MR, Stewart A, Ayers D, Brody EN, Williams SA, Levin S, Black B, Harbut M, Carbone M, et al: Early detection of malignant pleural mesothelioma in asbestosexposed individuals with a noninvasive proteomics-based surveillance tool. PLoS One 7: e46091, 2012.

3. Kishimoto T, Ozaki S, Kato K, Nishi H and Genba K: Malignant pleural mesothelioma in parts of Japan in relationship to asbestos exposure. Ind Health 42: 435-439, 2004.

4. Takahashi H, Harada M, Maehara S and Kato H: Localized malignant mesothelioma of the pleura. Ann Thorac Cardiovasc Surg 13: 262-266, 2007.

5. Hodgson JT, McElvenny DM, Darnton AJ, Price MJ and Peto J: The expected burden of mesothelioma mortality in Great Britain from 2002 to 2050. Br J Cancer 92: 587-593, 2005.

6. Joshi TK and Gupta RK: Asbestos-related morbidity in India. Int J Occup Environ Health 9: 249-253, 2003.

7. Lucchi M, Chella A, Melfi F, Dini P, Tibaldi C, Fontanini G and Mussi A: Four-modality therapy in malignant pleural mesothelioma: A phase II study. J Thorac Oncol 2: 237-242, 2007.

8. Stahel RA, Weder W, Lievens Y and Felip E; ESMO Guidelines Working Group: Malignant pleural mesothelioma: ESMO Clinical Practice Guidelines for diagnosis, treatment and follow-up. Ann Oncol 21 (Suppl 5): v126-v128, 2010.

9. Nasreen N, Khodayari N and Mohammed KA: Advances in malignant pleural mesothelioma therapy: Targeting EphA2 a novel approach. Am J Cancer Res 2: 222-234, 2012.

10. Steele JP and Klabatsa A: Chemotherapy options and new advances in malignant pleural mesothelioma. Ann Oncol 16: 345-351, 2005.

11. Ceresoli GL, Gridelli C and Santoro A: Multidisciplinary treatment of malignant pleural mesothelioma. Oncologist 12 : 850-863, 2007.

12. Katirtzoglou N, Gkiozos I, Makrilia N, Tsaroucha E, Rapti A, Stratakos G, Fountzilas G and Syrigos KN: Carboplatin plus pemetrexed as first-line treatment of patients with malignant pleural mesothelioma: A phase II study. Clin Lung Cancer 11: 30-35, 2010.

13. Sorensen JB, Sundstrom S, Perell K and Thielsen AK: Pemetrexed as second-line treatment in malignant pleural mesothelioma after platinum-based first-line treatment. J Thorac Oncol 2: 147-152, 2007.

14. Hanauske AR, Chen V, Paoletti P and Niyikiza C: Pemetrexed disodium: A novel antifolate clinically active against multiple solid tumors. Oncologist 6: 363-373, 2001.

15. Marangolo $M$ and Vertogen B: Pemetrexed and malignant pleural mesothelioma. Ann Oncol 17 (Suppl 5): v103-v105, 2006.
16. Scagliotti GV, Shin DM, Kindler HL, Vasconcelles MJ, Keppler U, Manegold C, Burris H, Gatzemeier U, Blatter J, Symanowski JT, et al: Phase II study of pemetrexed with and without folic acid and vitamin B12 as front-line therapy in malignant pleural mesothelioma. J Clin Oncol 21: 1556-1561, 2003.

17. Ozasa H, Oguri T, Uemura T, Miyazaki M, Maeno K, Sato S and Ueda R: Significance of thymidylate synthase for resistance to pemetrexed in lung cancer. Cancer Sci 101: 161-166, 2010.

18. Zhang D, Ochi N, Takigawa N, Tanimoto Y, Chen Y, Ichihara E, Hotta K, Tabata M, Tanimoto M and Kiura K: Establishment of pemetrexed-resistant non-small cell lung cancer cell lines. Cancer Lett 309: 228-235, 2011.

19. Milhavet O, Gary DS and Mattson MP: RNA interference in biology and medicine. Pharmacol Rev 55: 629-648, 2003.

20. Shirasaki T, Maruya S, Mizukami H, Kakehata S, Kurotaki H, Yagihashi $\mathrm{S}$ and Shinkawa $\mathrm{H}$ : Effects of small interfering RNA targeting thymidylate synthase on survival of ACC 3 cells from salivary adenoid cystic carcinoma. BMC Cancer 8: 348, 2008.

21. Jeang KT: RNAi in the regulation of mammalian viral infections. BMC Biol 10: 58, 2012.

22. John M, Constien R, Akinc A, Goldberg M, Moon Y-A, Spranger M, Hadwiger P, Soutschek J, Vornlocher H-P, Manoharan M, et al: Effective RNAi-mediated gene silencing without interruption of the endogenous microRNA pathway. Nature 449: 745-747, 2007.

23. Nakamura K, Abu Lila AS, Matsunaga M, Doi Y, Ishida T and Kiwada H: A double-modulation strategy in cancer treatment with a chemotherapeutic agent and siRNA. Mol Ther 19: 2040-2047, 2011.

24. Leung RK and Whittaker PA: RNA interference: From gene silencing to gene-specific therapeutics. Pharmacol Ther 107: 222-239, 2005.

25. Abu Lila AS, Matsumoto H, Doi Y, Nakamura H, Ishida T and Kiwada H: Tumor-type-dependent vascular permeability constitutes a potential impediment to the therapeutic efficacy of liposomal oxaliplatin. Eur J Pharm Biopharm 81: 524-531, 2012.

26. Bartlett GR: Colorimetric assay methods for free and phosphorylated glyceric acids. J Biol Chem 234: 469-471, 1959.

27. Kviecinski MR, Felipe KB, Schoenfelder T, de Lemos Wiese LP, Rossi MH, Gonçalez E, Felicio JD, Filho DW and Pedrosa RC: Study of the antitumor potential of Bidens pilosa (Asteraceae) used in Brazilian folk medicine. J Ethnopharmacol 117: 69-75, 2008.

28. Abu Lila AS, Kizuki S, Doi Y, Suzuki T, Ishida T and Kiwada H: Oxaliplatin encapsulated in PEG-coated cationic liposomes induces significant tumor growth suppression via a dual-targeting approach in a murine solid tumor model. J Control Release 137: 8-14, 2009.

29. Sharma P, Brown S, Walter G, Santra S and Moudgil B: Nanoparticles for bioimaging. Adv Colloid Interface Sci 123-126: 471-485, 2006.

30. Takezawa K, Okamoto I, Okamoto W, Takeda M, Sakai K, Tsukioka S, Kuwata K, Yamaguchi H, Nishio K and Nakagawa K: Thymidylate synthase as a determinant of pemetrexed sensitivity in non-small cell lung cancer. Br J Cancer 104: 1594-1601, 2011.

31. Shintani Y, Ohta M, Hirabayashi H, Tanaka H, Iuchi K, Nakagawa K, Maeda H, Kido T, Miyoshi S and Matsuda H: New prognostic indicator for non-small-cell lung cancer, quantitation of thymidylate synthase by real-time reverse transcription polymerase chain reaction. Int J Cancer 104: 790-795, 2003.

32. Hashimoto H, Ozeki Y, Sato M, Obara K, Matsutani N, Nakagishi Y, Ogata T and Maehara T: Significance of thymidylate synthase gene expression level in patients with adenocarcinoma of the lung. Cancer 106: 1595-1601, 2006.

33. Hannon GJ: RNA interference. Nature 418: 244-251, 2002.

34. Huang C, Li M, Chen C and Yao Q: Small interfering RNA therapy in cancer: Mechanism, potential targets, and clinical applications. Expert Opin Ther Targets 12: 637-645, 2008.

35. Tabach Y, Billi AC, Hayes GD, Newman MA, Zuk O, Gabel H, Kamath R, Yacoby K, Chapman B, Garcia SM, et al: Identification of small RNA pathway genes using patterns of phylogenetic conservation and divergence. Nature 493: 694-698, 2013.

36. Chen HY, Wang JM, Wang HY, Zhang Y, Liu W, Pan L, Wang W, Chen S, Jin W and Wang L: Effect of short hairpin RNA-induced CXCR4 silence on ovarian cancer cell. Biomed Pharmacother 66: 549-553, 2012.

37. Castanotto D and Rossi JJ: The promises and pitfalls of RNA-interference-based therapeutics. Nature 457: 426-433, 2009. 
38. Terasawa K, Shimizu K and Tsujimoto G: Synthetic pre-miRNAbased shRNA as potent RNAi triggers. J Nucleic Acids 131579: 2011, 2011.

39. Jackson AL and Linsley PS: Recognizing and avoiding siRNA off-target effects for target identification and therapeutic application. Nat Rev Drug Discov 9: 57-67, 2010.

40. Iwahori K, Serada S, Fujimoto M, Nomura S, Osaki T, Lee CM, Mizuguchi H, Takahashi T, Ripley B, Okumura M, et al: Overexpression of SOCS3 exhibits preclinical antitumor activity against malignant pleural mesothelioma. Int J Cancer 129 1005-1017, 2011

41. Sterman DH, Recio A, Carroll RG, Gillespie CT, Haas A, Vachani A, Kapoor V, Sun J, Hodinka R, Brown JL, et al: A phase I clinical trial of single-dose intrapleural IFN-beta gene transfer for malignant pleural mesothelioma and metastatic pleural effusions: High rate of antitumor immune responses. Clin Cancer Res 13: 4456-4466, 2007.
42. Sterman DH, Recio A, Vachani A, Sun J, Cheung L, DeLong P, Amin KM, Litzky LA, Wilson JM, Kaiser LR et al: Long-term follow-up of patients with malignant pleural mesothelioma receiving high-dose adenovirus herpes simplex thymidine kinase/ganciclovir suicide gene therapy. Clin Cancer Res 11: 7444-7453, 2005

43. Storch K and Cordes N: Focal adhesion-chromatin linkage controls tumor cell resistance to radio- and chemotherapy. Chemother Res Pract 2012: 319287, 2012. 